\title{
Anti-cancer Properties of Green Tea Probed Viaquantum Mechanics Calculations
}

\begin{abstract}
AZIN CHITSAZAN
Department of Chemistry, Science and Research Branch, Islamic Azad University, Tehran, Iran.

${ }^{*}$ Correspondign author E-mail: chitsazan63@gmail.com
\end{abstract}

http://dx.doi.org/10.13005/ojc/310147

(Received: November 30, 2014; Accepted: January 10, 2015)

\begin{abstract}
Tea, from the plant camellia sinensis, is consumed in different parts of the world as green, black or oolong tea. Among all of these, however, the most significant effects on human health have been observed with the consumption of green tea. Green tea contains polyphenols, which include flavanols, flavandiols, flavonoids, and phenolic acids. Most of the green tea polyphenols (GTPs) are flavonols, commonly known as catechins. There are four kinds of catechins mainly find in green tea: epicatechin, epigallocatechin, epicatechin-3-gallate, and EGCG. Green tea catechins have demonstrated significant antioxidant, anticarcinogenic, anti-inflammatory, thermogenic, probiotic, and antimicrobial properties in numerous human, animal, and in vitro studies. In the present study, four type catechins of green tea were studied. For each catechin ab initio method was employed for calculations and related parameters were computed.
\end{abstract}

Key words:DFT calculation, green tea,catechin, polyphenol, EGCG, antioxidant, anticarcinogenic.

\section{INTRODUCTION}

Tea is the most consumed drink in the world after water. Unlike black and oolong tea, green tea production does not involve oxidation of young tea leaves ${ }^{1-3}$.

Green tea is produced from steaming fresh leaves at high temperatures, thereby inactivating the oxidizing enzymes and leaving the polyphenol content intact ${ }^{4-8}$.
The polyphenols found in tea are more commonly known as flavanolsarcatechins, and comprise 30-40 percent of extractable solids of dried green tea leaves. The main catechins in green tea are epicatechin, epicatechin-3-gallate, epigallocatechin, and epigallocatechin-3-gallate (EGCG), with the latter being the highest in concentration ${ }^{9-13}$.

Green tea polyphenols have
demonstrated significant antioxidant,


Table 1: Results of optimize calculations for four catechins in green tea

\begin{tabular}{lcccccc}
\hline Molecule & $\begin{array}{c}\text { Energy } \\
\text { (Kcal/mol) }\end{array}$ & $\begin{array}{c}\text { Energy Gap } \\
\text { (Kcal/mol) }\end{array}$ & $\begin{array}{c}\text { Dipole } \\
\text { moment }\end{array}$ & $\begin{array}{c}\text { Quadrupole } \\
\text { moment }\end{array}$ & $\begin{array}{c}\text { Determinant of } \\
\text { condensed to } \\
\text { atoms }\end{array}$ & $\begin{array}{c}\text { Determinant of } \\
\text { Distance } \\
\text { matrix }\end{array}$ \\
\hline EC & -647167.1897 & -126.9633 & 3.1259 & -1508580 & $2.36359 \times 10^{11}$ & $1.55372 \times 10^{11}$ \\
EGC & -694366.2589 & -130.4898 & 4.0027 & -1578059 & $1.1503 \times 10^{12}$ & 0 \\
ECG & -1004872.918 & -102.2144 & 3.6648 & -5175210 & $1.51545 \times 10^{18}$ & $-3.98814 \times 10^{15}$ \\
EGCG & -1052067.736 & -106.9709 & 6.6295 & -5568137 & - & - \\
\hline
\end{tabular}

Table 2: Results of NMR calculations for(-)-epicatechin (EC)

\begin{tabular}{|c|c|c|c|c|c|}
\hline $\begin{array}{l}\text { Number } \\
\text { of Atom }\end{array}$ & Atom & Isotropic & Anisotropy & $\begin{array}{c}\text { Determinant of } \\
\text { Magnetic Shielding Tensor }\end{array}$ & $\begin{array}{l}\text { Determinant of } \\
\text { Eigen value }\end{array}$ \\
\hline 1 & $\mathrm{C}$ & 40.7933 & 129.4848 & -131534.6859 & -132187.3863 \\
\hline 2 & $\mathrm{C}$ & 101.1178 & 81.4917 & 749999.9317 & 749900.18 \\
\hline 3 & $\mathrm{C}$ & 41.682 & 124.4068 & -152111.1946 & -152254.7644 \\
\hline 4 & $\mathrm{C}$ & 98.2606 & 105.3572 & 618971.935 & 617123.4746 \\
\hline 5 & $\mathrm{C}$ & 40.3898 & 119.5755 & -120581.4747 & -121553.1794 \\
\hline 6 & $\mathrm{C}$ & 99.4788 & 96.0498 & 661610.9959 & 661291.5423 \\
\hline 7 & $\mathrm{O}$ & 214.1367 & 53.5446 & 9450047.009 & 9443779.294 \\
\hline 8 & $\mathrm{O}$ & 222.0782 & 73.8408 & 10461582.97 & 10407456.39 \\
\hline 9 & $\mathrm{C}$ & 161.8983 & 16.4821 & 4221606.349 & 4220733.264 \\
\hline 10 & $\mathrm{C}$ & 120.888 & 35.8661 & 1722200.697 & 1713626.209 \\
\hline 11 & $\mathrm{C}$ & 109.2673 & 32.904 & 1268005.561 & 1267203.469 \\
\hline 12 & $\mathrm{O}$ & 215.3092 & 78.6997 & 9240810.61 & 9165813.035 \\
\hline 13 & $\mathrm{C}$ & 83.8394 & 109.1486 & 135341.9435 & 131769.2325 \\
\hline 14 & $\mathrm{C}$ & 67.379 & 150.6034 & -70446.14768 & -72893.33234 \\
\hline 15 & $\mathrm{C}$ & 75.7056 & 160.0222 & -153597.7881 & -153847.8002 \\
\hline 16 & $\mathrm{C}$ & 78.9179 & 137.6591 & 49548.04689 & 47591.7128 \\
\hline 17 & $\mathrm{C}$ & 51.6981 & 118.1888 & -25234.30243 & -28346.03287 \\
\hline 18 & $\mathrm{C}$ & 55.7289 & 110.455 & -14237.44592 & -23269.86969 \\
\hline 19 & $\mathrm{O}$ & 242.7435 & 37.6084 & 14057512.95 & 14048991.28 \\
\hline 20 & $\mathrm{O}$ & 237.3366 & 93.0112 & 12686727.08 & 12634889.21 \\
\hline 21 & $\mathrm{O}$ & 281.7297 & 78.6015 & 21839351.01 & 21763132.24 \\
\hline 22 & $\mathrm{H}$ & 26.9099 & 4.8073 & 19130.43033 & 19130.05133 \\
\hline 23 & $\mathrm{H}$ & 26.4725 & 6.6187 & 18050.67965 & 18048.18389 \\
\hline 24 & $\mathrm{H}$ & 28.7916 & 12.6973 & 22036.45096 & 22029.18026 \\
\hline 25 & $\mathrm{H}$ & 28.3778 & 11.2004 & 20559.56103 & 20549.31111 \\
\hline 26 & $\mathrm{H}$ & 29.7656 & 7.1211 & 25573.39622 & 25558.2547 \\
\hline 27 & $\mathrm{H}$ & 30.0048 & 6.6191 & 26405.99076 & 26383.14398 \\
\hline 28 & $\mathrm{H}$ & 28.6196 & 2.3067 & 23414.05176 & 23383.86483 \\
\hline 29 & $\mathrm{H}$ & 27.8962 & 5.8829 & 21344.96671 & 21343.94067 \\
\hline 30 & $\mathrm{H}$ & 26.0539 & 8.5437 & 17044.42103 & 17039.40225 \\
\hline 31 & $\mathrm{H}$ & 25.0369 & 9.2777 & 14899.52311 & 14898.21926 \\
\hline 32 & $\mathrm{H}$ & 25.3491 & 5.0347 & 15942.47712 & 15938.84851 \\
\hline 33 & $\mathrm{H}$ & 29.0284 & 13.5478 & 22369.32596 & 22348.95548 \\
\hline 34 & $\mathrm{H}$ & 27.3461 & 12.1463 & 17802.84656 & 17801.49197 \\
\hline 35 & $\mathrm{H}$ & 31.505 & 16.669 & 27807.03155 & 27649.59731 \\
\hline
\end{tabular}




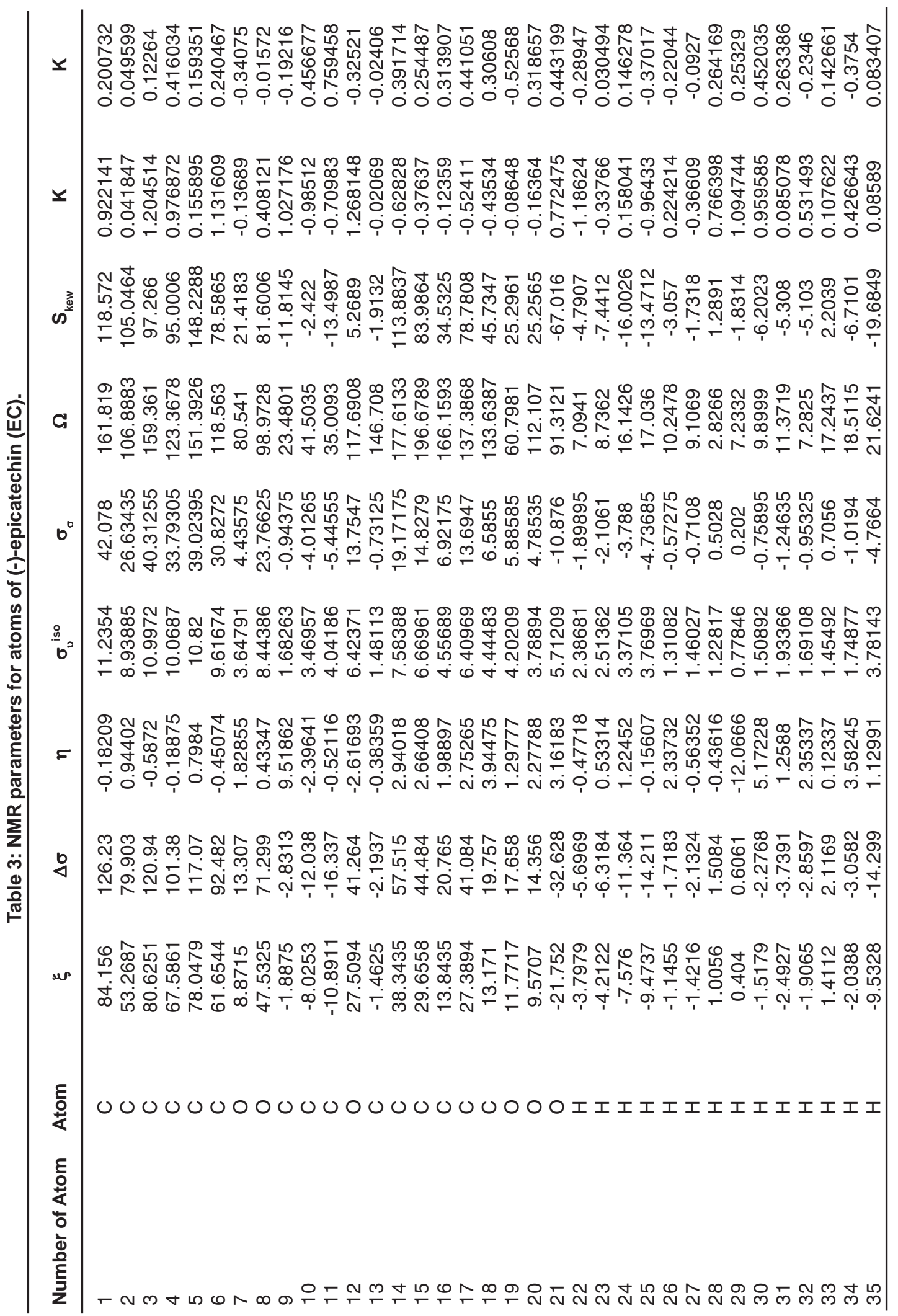


Table 4: Results of NMR calculations for (-)-epigallocatechin (EGC)

\begin{tabular}{|c|c|c|c|c|c|}
\hline $\begin{array}{l}\text { Number of } \\
\text { Atom }\end{array}$ & Atom & Isotropic & Anisotropy & $\begin{array}{l}\text { Determinant of Magnetic } \\
\text { Shielding Tensor }\end{array}$ & $\begin{array}{l}\text { Determinant of } \\
\text { Eigen value }\end{array}$ \\
\hline 1 & $\mathrm{C}$ & 40.8019 & 129.4337 & -131468.2548 & -132109.6327 \\
\hline 2 & $\mathrm{C}$ & 101.1489 & 81.4339 & 751314.675 & 751212.2715 \\
\hline 3 & C & 41.7131 & 124.2695 & -152261.0107 & -152407.9893 \\
\hline 4 & C & 98.2962 & 105.7753 & 617607.2076 & 615674.268 \\
\hline 5 & C & 40.4762 & 119.3192 & -120540.4738 & -121470.4712 \\
\hline 6 & C & 99.3432 & 96.1534 & 657786.1135 & 657460.7632 \\
\hline 7 & O & 214.1095 & 53.6071 & 9447285.642 & 9440995.986 \\
\hline 8 & O & 222.082 & 73.5106 & 10464384.51 & 10411199.43 \\
\hline 9 & $\mathrm{C}$ & 161.9189 & 16.7679 & 4221685.215 & 4220717.894 \\
\hline 10 & C & 121.4412 & 36.0943 & 1746115.875 & 1737920.775 \\
\hline 11 & $\mathrm{C}$ & 109.415 & 32.5108 & 1274159.56 & 1273308.594 \\
\hline 12 & O & 216.1166 & 80.7238 & 9325726.234 & 9246871.596 \\
\hline 13 & C & 92.4905 & 96.9895 & 355343.3305 & 351557.0033 \\
\hline 14 & C & 65.6881 & 155.1177 & -80826.25932 & -84441.42628 \\
\hline 15 & C & 88.3958 & 130.7809 & 233168.3214 & 230188.5697 \\
\hline 16 & $\mathrm{C}$ & 49.6752 & 118.0147 & -27097.08213 & -31945.97089 \\
\hline 17 & C & 65.0882 & 97.6628 & 131362.7578 & 131304.7711 \\
\hline 18 & $\mathrm{C}$ & 55.4935 & 108.5991 & -1705.637668 & -12467.12478 \\
\hline 19 & O & 243.1531 & 36.5631 & 14126374.47 & 14117995.46 \\
\hline 20 & O & 264.2844 & 85.8033 & 17851122.03 & 17824969.09 \\
\hline 21 & O & 234.4268 & 97.6819 & 12142554.41 & 12089030.39 \\
\hline 22 & O & 280.8084 & 78.6167 & 21625261.39 & 21543683.18 \\
\hline 23 & $\mathrm{H}$ & 26.9056 & 4.7901 & 19115.63886 & 19115.18925 \\
\hline 24 & $\mathrm{H}$ & 26.4412 & 6.7028 & 17974.30364 & 17971.87533 \\
\hline 25 & $\mathrm{H}$ & 28.7934 & 12.7335 & 22032.02381 & 22024.99256 \\
\hline 26 & $\mathrm{H}$ & 28.3798 & 11.1724 & 20558.6524 & 20548.90834 \\
\hline 27 & $\mathrm{H}$ & 29.7438 & 7.1605 & 25504.48508 & 25490.06658 \\
\hline 28 & $\mathrm{H}$ & 30.0328 & 6.5941 & 26474.82355 & 26451.09014 \\
\hline 29 & $\mathrm{H}$ & 28.5931 & 2.2897 & 23351.60052 & 23320.64108 \\
\hline 30 & $\mathrm{H}$ & 27.9385 & 5.6683 & 21457.45614 & 21456.58277 \\
\hline 31 & $\mathrm{H}$ & 26.5138 & 7.8997 & 18034.92192 & 18029.56595 \\
\hline 32 & $\mathrm{H}$ & 25.4382 & 9.8619 & 15572.96794 & 15568.46717 \\
\hline 33 & $\mathrm{H}$ & 29.02 & 13.8912 & 22268.71786 & 22249.01053 \\
\hline 34 & $\mathrm{H}$ & 27.8835 & 14.2057 & 18641.81058 & 18635.7062 \\
\hline 35 & $\mathrm{H}$ & 27.2655 & 12.5265 & 17574.15693 & 17573.94869 \\
\hline 36 & $\mathrm{H}$ & 31.3822 & 17.1506 & 27243.58921 & 27092.09421 \\
\hline
\end{tabular}




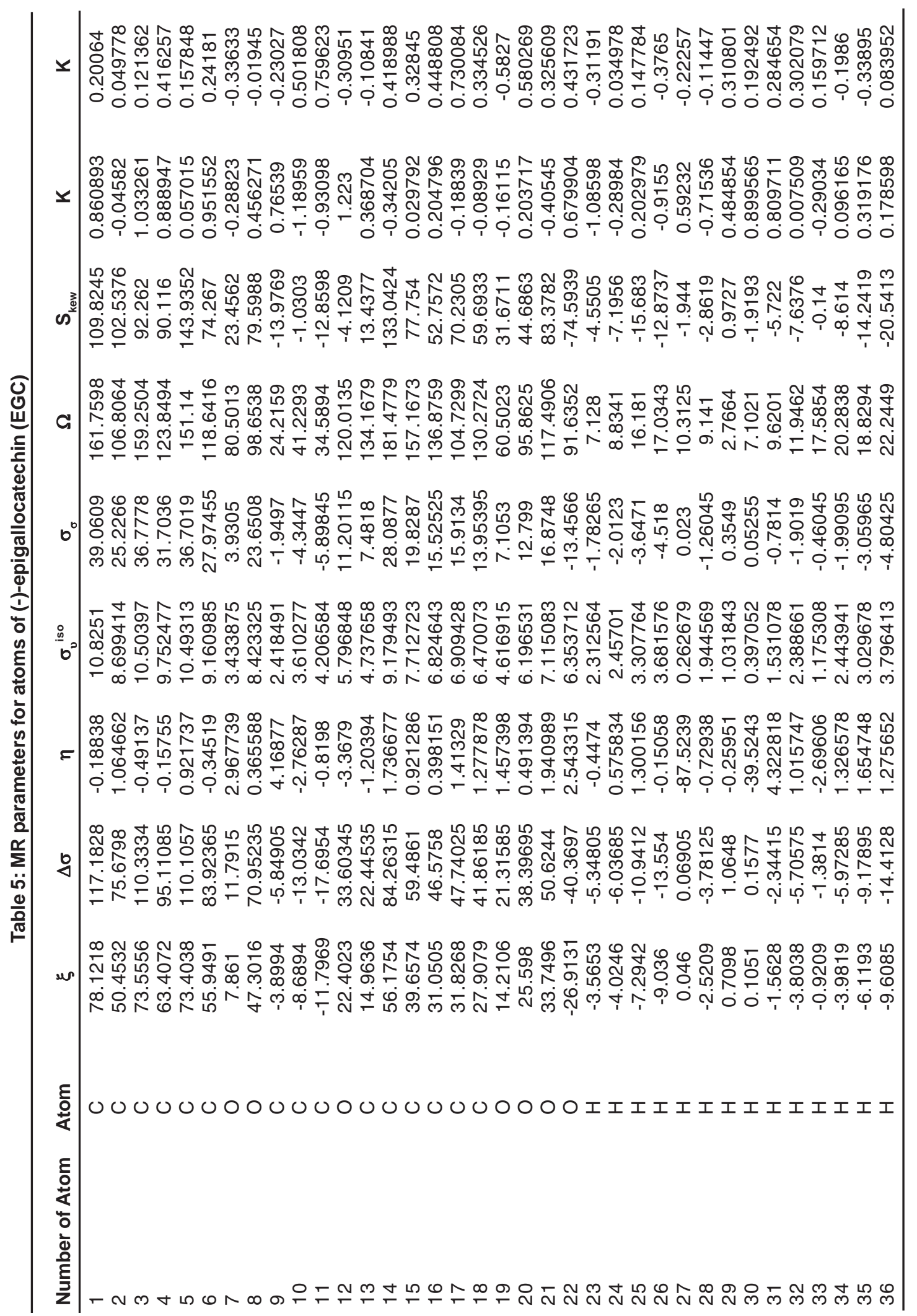


Table 6: Results of NMR calculations for (-)-epicatechin-3-gallate(ECG)

\begin{tabular}{|c|c|c|c|c|c|}
\hline $\begin{array}{l}\text { Number of } \\
\text { Atom }\end{array}$ & Atom & Isotropic & Anisotropy & $\begin{array}{l}\text { Determinant of Magnetic } \\
\text { Shielding Tensor }\end{array}$ & $\begin{array}{l}\text { Determinant of } \\
\text { Eigen value }\end{array}$ \\
\hline 1 & C & 40.4146 & 130.0009 & -128615 & -129298 \\
\hline 2 & C & 100.8331 & 81.9869 & 741250.4 & 741138.7 \\
\hline 3 & C & 41.7913 & 124.3387 & -153638 & -153693 \\
\hline 4 & C & 99.2153 & 103.3459 & 650003.2 & 648009.2 \\
\hline 5 & C & 40.915 & 119.5993 & -126026 & -127067 \\
\hline 6 & C & 99.2005 & 96.3905 & 652721.2 & 652351.4 \\
\hline 7 & 0 & 213.5399 & 53.3362 & 9356683 & 9350524 \\
\hline 8 & 0 & 221.7059 & 73.539 & 10412724 & 10360978 \\
\hline 9 & C & 161.8432 & 18.892 & 4216965 & 4215851 \\
\hline 10 & $\mathrm{C}$ & 111.9613 & 50.4775 & 1313604 & 1307693 \\
\hline 11 & C & 112.7245 & 38.8766 & 1381136 & 1378868 \\
\hline 12 & 0 & 217.6278 & 74.2272 & 9588378 & 9507721 \\
\hline 13 & C & 76.6871 & 131.5557 & 19844.72 & 16156.17 \\
\hline 14 & C & 69.8138 & 147.6212 & -58409.9 & -59125.5 \\
\hline 15 & C & 76.2222 & 161.6723 & -148429 & -149378 \\
\hline 16 & C & 81.184 & 135.6802 & 84354.25 & 81708.69 \\
\hline 17 & C & 50.9251 & 121.5665 & -30327.8 & -35385.4 \\
\hline 18 & C & 55.4857 & 109.741 & -26581.9 & -34220.8 \\
\hline 19 & 0 & 251.2283 & 40.5396 & 15622818 & 15483215 \\
\hline 20 & 0 & 239.1597 & 93.2347 & 13003724 & 12931497 \\
\hline 21 & 0 & 134.0707 & 145.1335 & -390236 & -520509 \\
\hline 22 & C & 73.6342 & 135.9389 & -30737.7 & -30908.9 \\
\hline 23 & C & 87.8734 & 125.3321 & 272625.8 & 271983.8 \\
\hline 24 & C & 52.5862 & 115.2903 & -7435.83 & -11267.4 \\
\hline 25 & C & 61.3147 & 103.8535 & 86001.26 & 85805.84 \\
\hline 26 & C & 54.2027 & 111.3854 & -21345.3 & -28559.7 \\
\hline 27 & C & 86.7742 & 116.5835 & 205590.6 & 204884 \\
\hline 28 & C & 26.4825 & 77.4218 & -280606 & -283156 \\
\hline 29 & 0 & -95.4139 & 579.7699 & 21461495 & 21461917 \\
\hline 30 & 0 & 242.1249 & 32.4512 & 13923375 & 13917987 \\
\hline 31 & 0 & 257.1364 & 98.1199 & 16228249 & 16212366 \\
\hline 32 & 0 & 236.9415 & 93.018 & 12635932 & 12574274 \\
\hline 33 & $\mathrm{H}$ & 26.8644 & 4.9382 & 19019.39 & 19018.64 \\
\hline 34 & $\mathrm{H}$ & 26.4448 & 6.8991 & 17956.37 & 17954.87 \\
\hline 35 & $\mathrm{H}$ & 28.7275 & 12.7808 & 21858.77 & 21851.09 \\
\hline 36 & $\mathrm{H}$ & 28.3537 & 10.9946 & 20603.79 & 20594.37 \\
\hline 37 & $\mathrm{H}$ & 29.4838 & 5.8049 & 24661.02 & 24656.26 \\
\hline 38 & $\mathrm{H}$ & 29.597 & 6.1079 & 25334.84 & 25314.18 \\
\hline 39 & $\mathrm{H}$ & 27.8979 & 7.5734 & 21136.99 & 21132.83 \\
\hline 40 & $\mathrm{H}$ & 27.4551 & 4.8791 & 20439.09 & 20427.47 \\
\hline 41 & $\mathrm{H}$ & 25.3289 & 8.1221 & 15747.41 & 15729.67 \\
\hline 42 & $\mathrm{H}$ & 25.2434 & 8.55 & 15354.62 & 15352.9 \\
\hline 43 & $\mathrm{H}$ & 25.6134 & 4.1254 & 16523.21 & 16517.52 \\
\hline 44 & $\mathrm{H}$ & 28.4687 & 13.8161 & 20973.78 & 20906.58 \\
\hline 45 & $\mathrm{H}$ & 27.2212 & 11.594 & 17655.35 & 17645.91 \\
\hline 46 & $\mathrm{H}$ & 25.9561 & 7.9531 & 16842.7 & 16840.41 \\
\hline 47 & $\mathrm{H}$ & 26.398 & 7.1822 & 17910.92 & 17902.09 \\
\hline 48 & $\mathrm{H}$ & 28.9066 & 13.5722 & 22041.84 & 22012.07 \\
\hline 49 & $\mathrm{H}$ & 27.603 & 13.8326 & 18078.81 & 18073.67 \\
\hline 50 & $\mathrm{H}$ & 27.4441 & 13.1068 & 17888.99 & 17888.06 \\
\hline
\end{tabular}




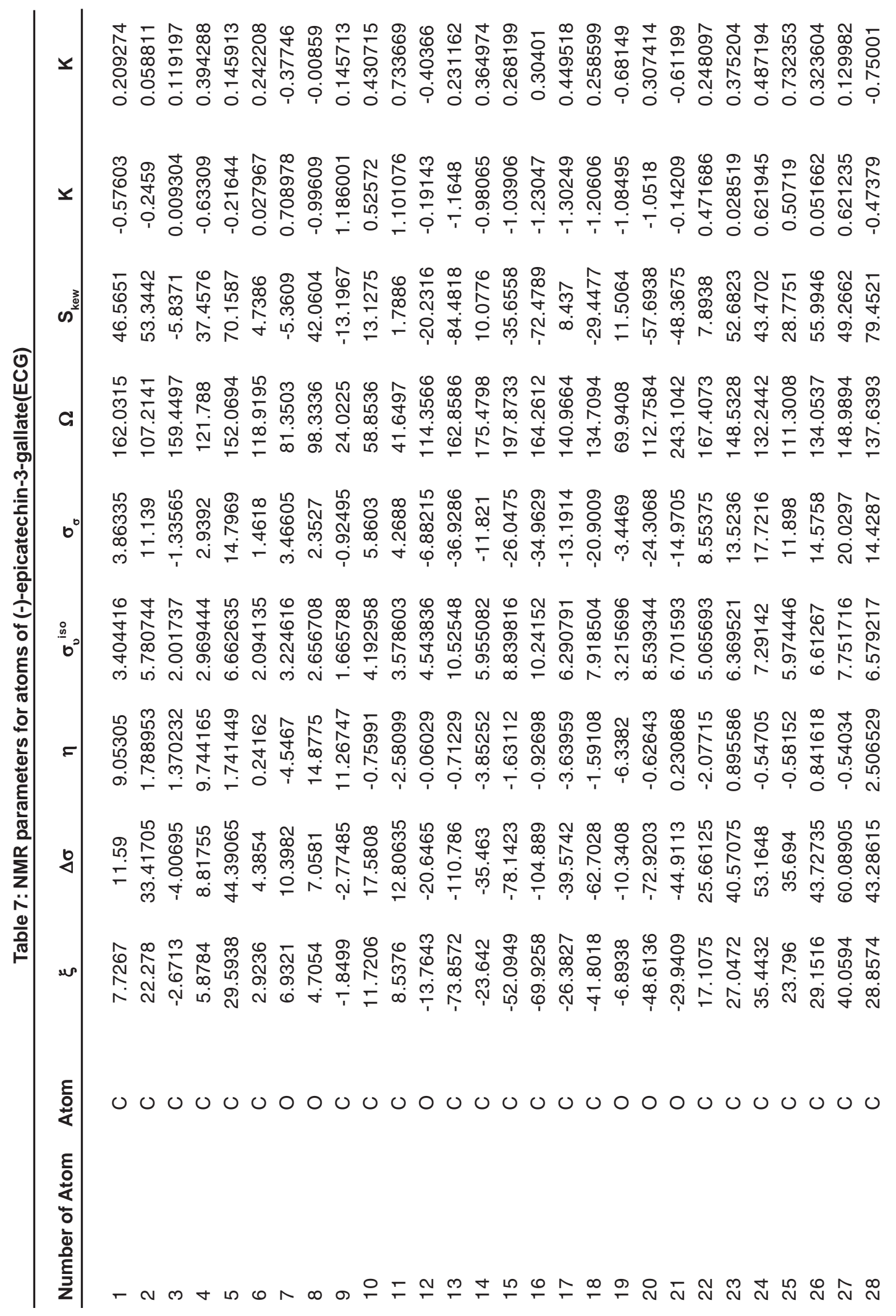


Ұ

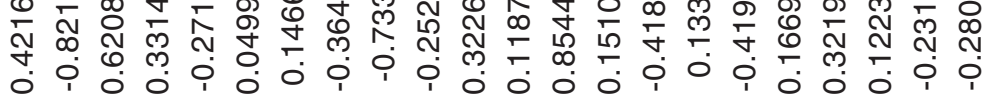

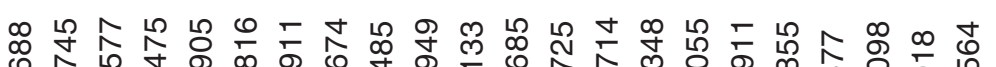

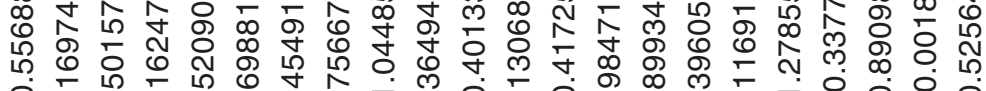

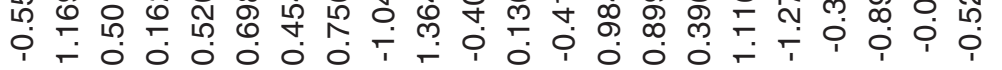

\& 잉 \& ठ্口

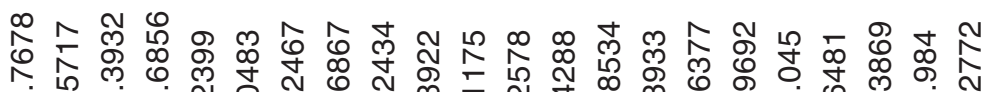
ヘ 仓

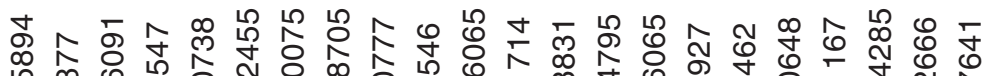

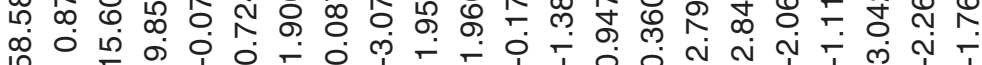

ํㅣㄴ

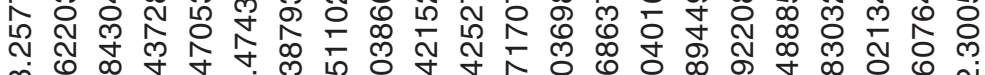

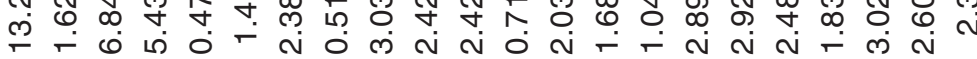

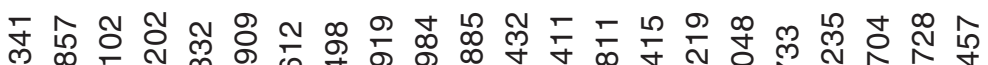

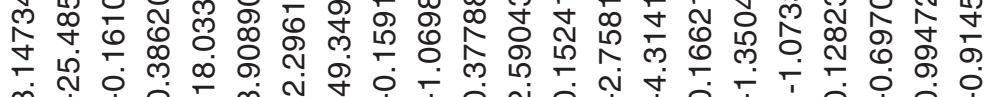
网

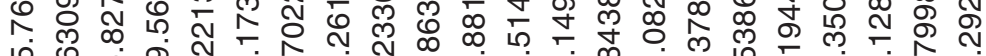

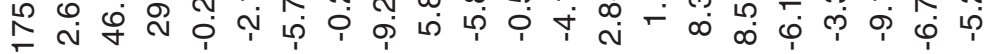

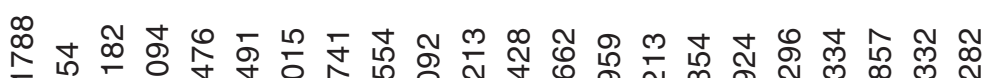
స. 卓 F-

O O O OIIIIIIIIIIIIIIIIII

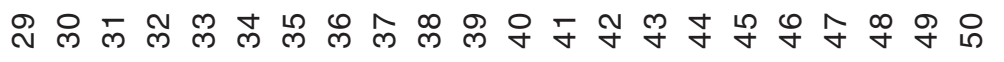


Table 8: Results of NMR calculations for(-)-epigallocatechin-3-gallate (EGCG)

\begin{tabular}{|c|c|c|c|c|c|}
\hline $\begin{array}{l}\text { Number of } \\
\text { Atom }\end{array}$ & Atom & Isotropic & Anisotropy & $\begin{array}{l}\text { Determinant of Magnetic } \\
\text { Shielding Tensor }\end{array}$ & $\begin{array}{l}\text { Determinant of } \\
\text { Eigen value }\end{array}$ \\
\hline 1 & $\mathrm{C}$ & 40.4524 & 129.862 & -128713 & -129210 \\
\hline 2 & $\mathrm{C}$ & 99.6995 & 97.8347 & 676158.5 & 676097.1 \\
\hline 3 & $\mathrm{C}$ & 40.8537 & 125.5443 & -150525 & -150577 \\
\hline 4 & $\mathrm{C}$ & 98.7956 & 103.4706 & 637057.1 & 635174.6 \\
\hline 5 & $\mathrm{C}$ & 41.047 & 119.6756 & -118159 & -119400 \\
\hline 6 & $\mathrm{C}$ & 100.695 & 80.7105 & 723196.7 & 722809.2 \\
\hline 7 & $\mathrm{O}$ & 213.9399 & 53.9164 & 9400224 & 9396174 \\
\hline 8 & $\mathrm{O}$ & 220.4964 & 75.2578 & 10202384 & 10154243 \\
\hline 9 & $\mathrm{C}$ & 160.964 & 18.3701 & 4151548 & 4150085 \\
\hline 10 & $\mathrm{C}$ & 113.5261 & 46.8777 & 1384243 & 1380054 \\
\hline 11 & $\mathrm{C}$ & 108.7181 & 29.8684 & 1254469 & 1252936 \\
\hline 12 & $\mathrm{O}$ & 208.8412 & 80.9209 & 8390864 & 8313384 \\
\hline 13 & $\mathrm{C}$ & 84.4214 & 114.0603 & 180000.3 & 175519.3 \\
\hline 14 & $\mathrm{C}$ & 59.3692 & 169.6506 & -128925 & -129874 \\
\hline 15 & $\mathrm{C}$ & 91.1127 & 127.0327 & 294166.5 & 289658.6 \\
\hline 16 & C & 46.6936 & 124.9664 & -61746.4 & -67766.5 \\
\hline 17 & $\mathrm{C}$ & 67.5615 & 77.3282 & 182209.5 & 182107.1 \\
\hline 18 & $\mathrm{C}$ & 46.7106 & 125.4811 & -52114.6 & -58828.3 \\
\hline 19 & $\mathrm{O}$ & 232.0068 & 88.4187 & 11894662 & 11819705 \\
\hline 20 & $\mathrm{O}$ & 293.5939 & 134.5975 & 23578633 & 23572063 \\
\hline 21 & $\mathrm{O}$ & 146.9444 & 139.5594 & 368775 & 232676.1 \\
\hline 22 & $\mathrm{C}$ & 72.5049 & 136.8501 & -45651.7 & -46046 \\
\hline 23 & $\mathrm{C}$ & 87.5227 & 125.3313 & 270600.4 & 269816 \\
\hline 24 & $\mathrm{C}$ & 52.3823 & 115.2445 & -8546.29 & -12662.3 \\
\hline 25 & $\mathrm{C}$ & 61.6343 & 103.0269 & 89737.44 & 89581.53 \\
\hline 26 & $\mathrm{C}$ & 54.2623 & 110.6945 & -19111.6 & -26408.3 \\
\hline 27 & $\mathrm{C}$ & 86.6519 & 116.3523 & 202793.9 & 202173.4 \\
\hline 28 & C & 29.034 & 73.9967 & -308911 & -311190 \\
\hline 29 & $\mathrm{O}$ & -113.032 & 608.4679 & 26112351 & 26162998 \\
\hline 30 & $\mathrm{O}$ & 242.1023 & 34.3492 & 13928407 & 13925118 \\
\hline 31 & $\mathrm{O}$ & 257.8886 & 96.3126 & 16400978 & 16385666 \\
\hline 32 & $\mathrm{O}$ & 236.9009 & 92.7637 & 12632903 & 12572243 \\
\hline 33 & $\mathrm{O}$ & 234.9932 & 86.0768 & 12395803 & 12319916 \\
\hline 34 & $\mathrm{H}$ & 26.375 & 4.6461 & 17990.46 & 17990.01 \\
\hline 35 & $\mathrm{H}$ & 27.0259 & 7.2398 & 19184.59 & 19182.48 \\
\hline 36 & $\mathrm{H}$ & 28.8042 & 11.8772 & 22132.24 & 22128.13 \\
\hline 37 & $\mathrm{H}$ & 28.3005 & 10.5617 & 20584.77 & 20573.15 \\
\hline 38 & $\mathrm{H}$ & 29.5952 & 6.026 & 25423.05 & 25397.77 \\
\hline 39 & $\mathrm{H}$ & 29.4205 & 6.287 & 24434.1 & 24432.15 \\
\hline 40 & $\mathrm{H}$ & 27.6734 & 7.3151 & 20627.26 & 20607.36 \\
\hline 41 & $\mathrm{H}$ & 27.7448 & 6.1196 & 21005.79 & 20999.58 \\
\hline 42 & $\mathrm{H}$ & 25.8174 & 7.5733 & 16683.84 & 16677.62 \\
\hline 43 & $\mathrm{H}$ & 26.0377 & 7.9109 & 17139.27 & 17134.88 \\
\hline 44 & $\mathrm{H}$ & 27.475 & 12.1494 & 18314.9 & 18302.09 \\
\hline 45 & $\mathrm{H}$ & 30.5854 & 18.0495 & 25722.42 & 25644.91 \\
\hline 46 & $\mathrm{H}$ & 25.796 & 8.0162 & 16510.66 & 16504.99 \\
\hline 47 & $\mathrm{H}$ & 26.2549 & 6.6232 & 17688.98 & 17686.58 \\
\hline 48 & $\mathrm{H}$ & 28.9466 & 13.7936 & 22118.84 & 22094.03 \\
\hline 49 & $\mathrm{H}$ & 27.6021 & 13.9758 & 18055.48 & 18050.28 \\
\hline 50 & $\mathrm{H}$ & 27.4034 & 13.024 & 17806.56 & 17805.54 \\
\hline 51 & $\mathrm{H}$ & 27.3753 & 12.8376 & 18048.34 & 18031.36 \\
\hline
\end{tabular}




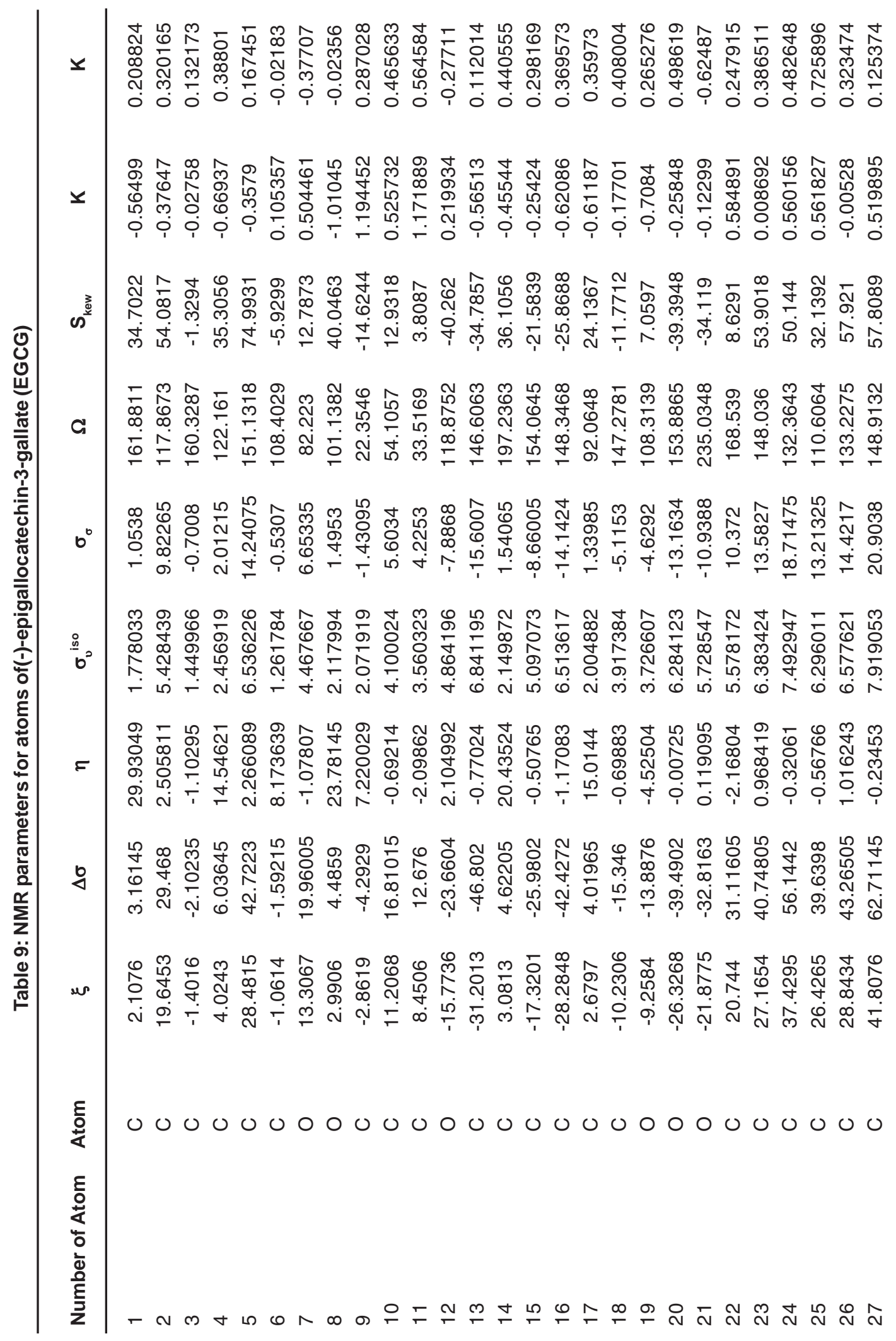




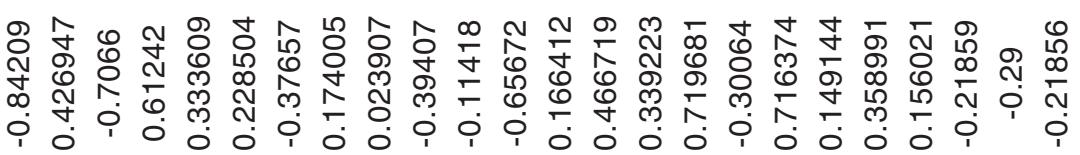
ল্ল

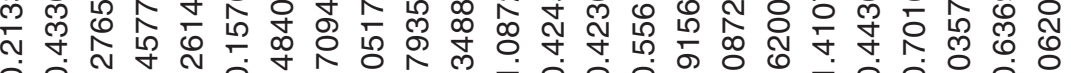
定

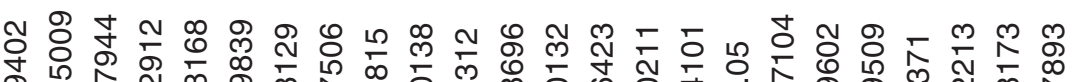
足

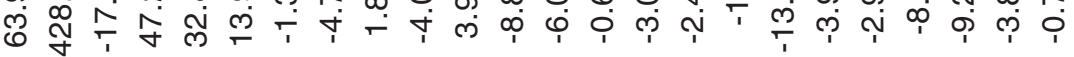

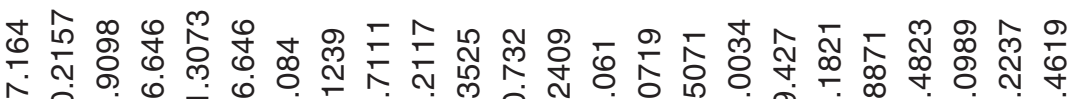

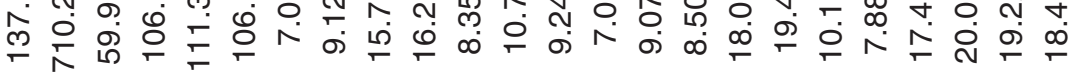

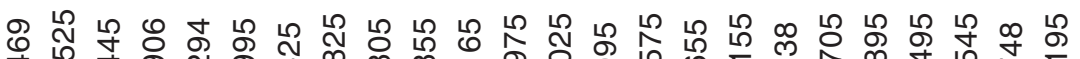

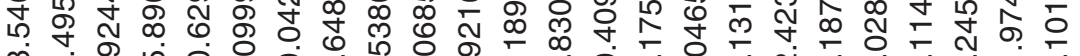
m)

\&

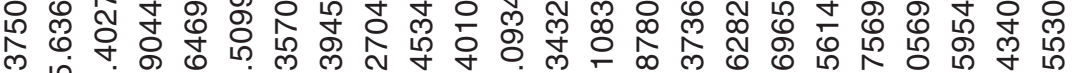
ט.

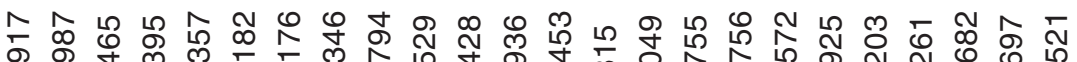

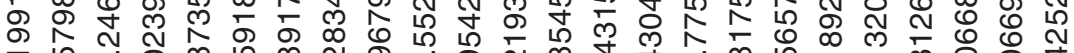
下 N

ஜ

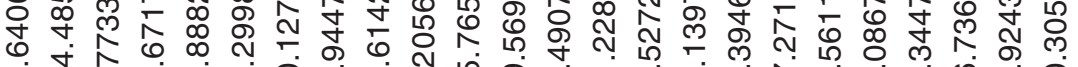

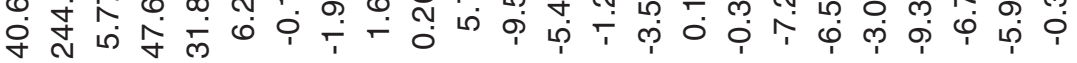

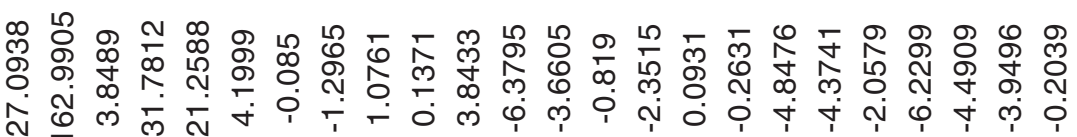

OOOOOOIIIIIIIIIIIIIIIIII

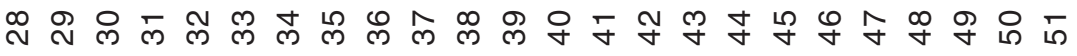


anticarcinogenic, anti-inflammatory, thermogenic, probitic, and antimicrobial properties in numerous human, animal, and in vitro studies ${ }^{14-18}$.

The anticarcinogenic properties of green tea polyphenols, mainly EGCG, are likely a result of inhibition of tumor initiation and promotion, induction of apoptosis, and inhibition of cell replication rates, thus retarding the growth and development of neoplasms ${ }^{19-22}$. Green tea polyphenols antioxidant potential is directly related to the combination of aromatic rings and hydroxyl groups that make up their structure, and is a result of binding and neutralization of free radicals by the hydroxyl groups $^{23-27}$.

The potential health effects of catechins depend not only on the amount consumed but on their bioavailability which appears to be very variable. In order to known the catechin bioavailability and metabolism, it is necessary to evaluate their biological activity within target tissues ${ }^{28-36}$.

Green tea is considered a dietary source of antioxidant nutrients: green tea is rich in polyphenols(catechins and gallic acid, particulary), but is also contains carotenoids, tocopherols, ascorbic acid (vitamin C), minerals such as $\mathrm{Cr}, \mathrm{Mn}$, Se or Zn, amd certain phytochemical compounds. These compounds could increase the green tea polyphenols (GTP) antioxidant potential. The antioxidant capacity of GTP has been assessed by several methods ${ }^{37-41}$.

For example, Cao et al..$^{42}$ using the oxygen radical absorbance capacity (ORAC) assay found that green tea has a much higher antioxidant activity against peroxyl radicals than vegetables such as garlic, kale, spinach and Brussels sprouts. LangleyEvans [43]found that the total antioxidant capacity of green tea is more potent than that of black tea.Saffari and Sadrzadeh ${ }^{44}$ investigated the antioxidant capacity of EGCG using erythrocyte membrane- bound ${ }^{45-51}$.

ATPases as model, and the results indicated that EGCG is a powerful antioxidant that is capable of protecting erythrocyte membranebound ATPases against oxidative stress. Several

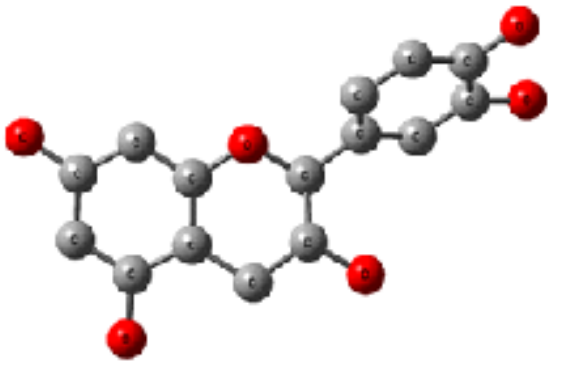

EC

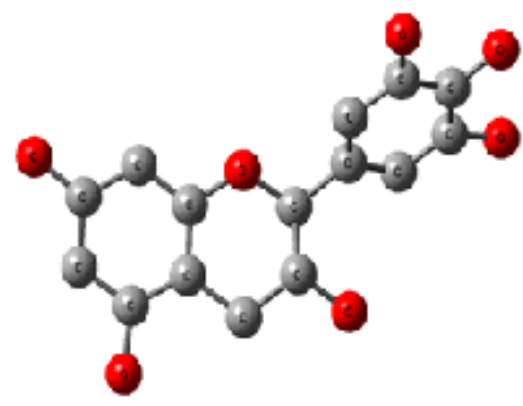

EGC

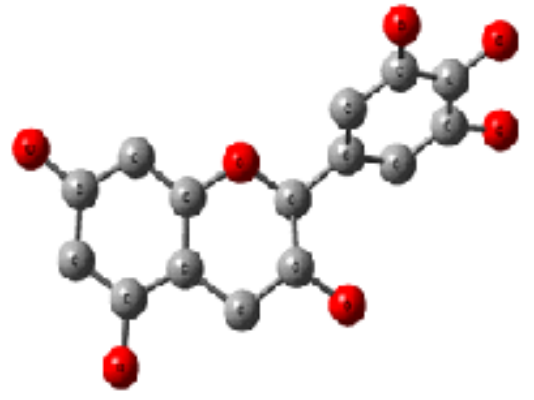

EGC

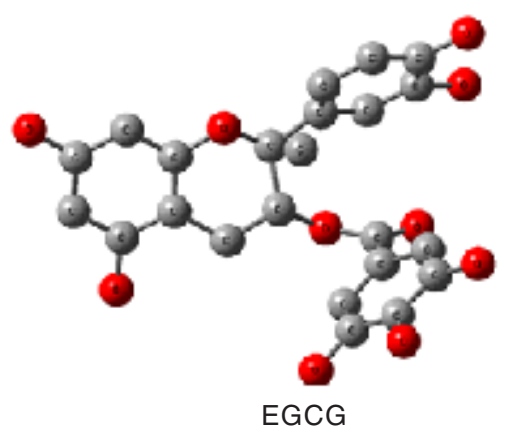

Fig. 1: Energetically favourable structure of four major catechins in green tea. EC, (-)-epicatechin; EGC, (-)-epigallocatechin; ECG, (-)-epicatechin3-gallate; EGCG, (-)-epigallocatechin-3-gallate 


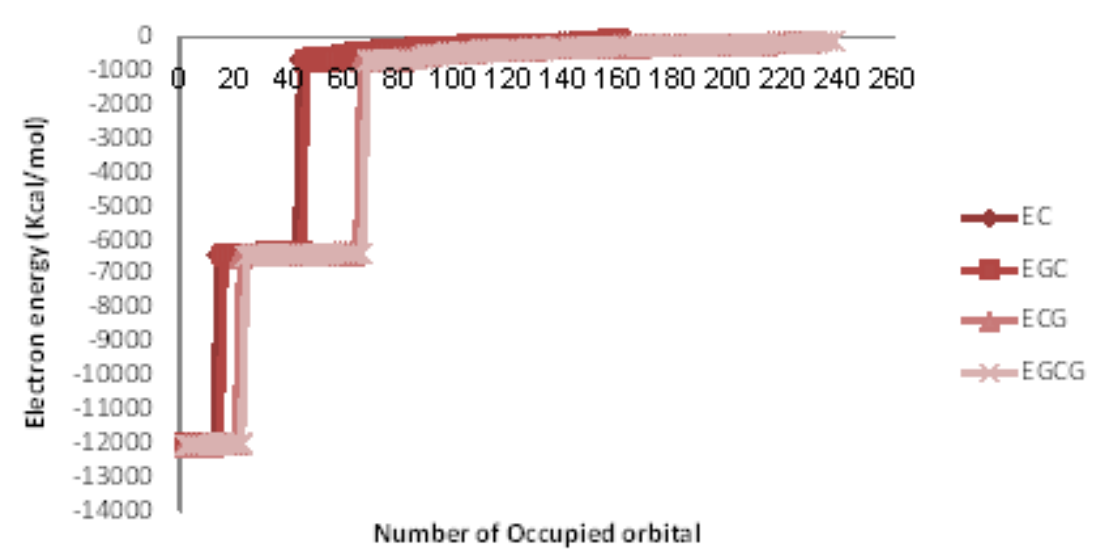

Fig. 2: Variation of electron energy in the occupied orbitals for EC, (-)-epicatechin; EGC, (-)-epigallocatechin; ECG, (-)-epicatechin-3-gallate; EGCG, (-)-epigallocatechin-3-gallate

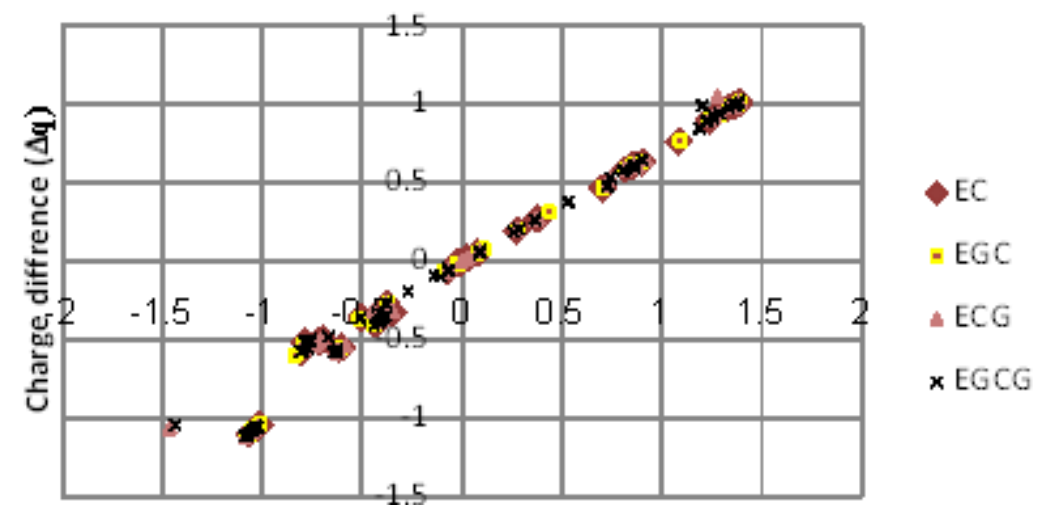

Fig. 3: Variation of charge difference $(\Delta q)$ as function of bond moment for $E C$, (-)-epicatechin; EGC, (-)-epigallocatechin; ECG, (-)-epicatechin-3-gallate; EGCG, (-)-epigallocatechin-3-gallate

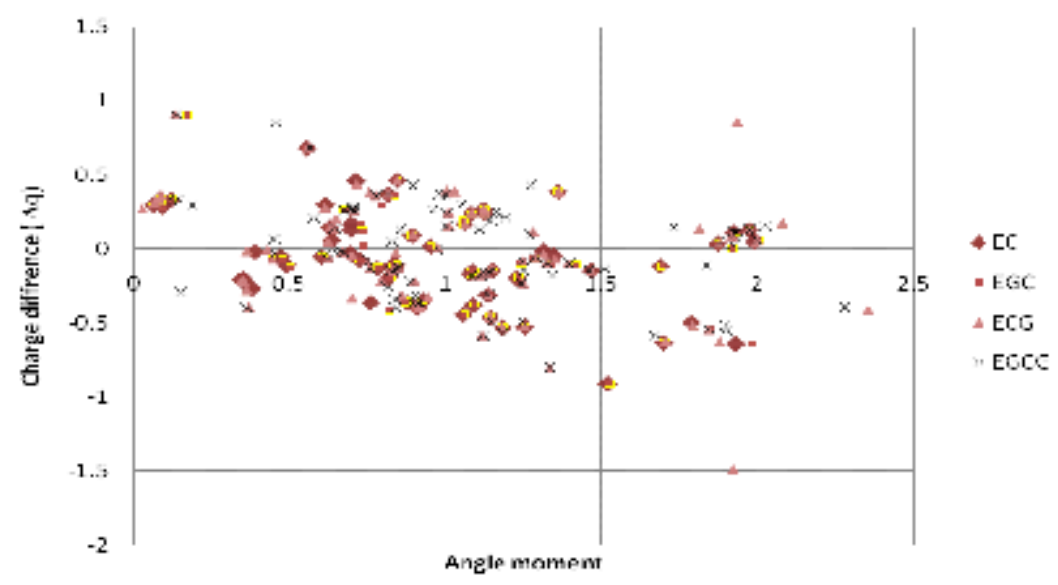

Fig. 4: Variation of charge difference $(\Delta q)$ as function of angle moment for $E C$, (-)-epicatechin; EGC, (-)-epigallocatechin; ECG, (-)-epicatechin-3-gallate; EGCG, (-)-epigallocatechin-3-gallate 
studies have shown that EGCG can act in vitroas an antioxidant by trapping proxyl radicals and inhibiting lipid peroxidation ${ }^{44,52}$. However, the antioxidant capacity of catechins determined in vitrois dependent upon the type of assay and it does not reflect factors such as bioavailability and metabolism ${ }^{53-59}$.

\section{Methods and Calculations}

Computational methods are well placed to a theoretical approach that the first-principle calculation to obtain various aspects of drug properties of green tea molecules ${ }^{60-63}$.

The first-principle quantum chemical calculations applied to investigation the nature of green tea molecules were performed in the density functional theory (DFT) system using the program package Gaussian 03. At first, the geometry of the molecules was optimized ${ }^{64-66}$.

In the all calculations molecule were treated with $6-31 \mathrm{G}^{*}$ basis set, carried out by B3LYP function 67,68 .

All these cases have been taken into account for several parameters such as energy, dipole moment, quadrupole moment, energy gap, electron energy and etc eq-71. $^{6}$.

As a result, energetically favourable structure of four molecules showed on figure 1 .

NMR calculations also were performed and results were obtained according to output files data.NMR parameters were achieved by following equations:

$$
\xi=\sigma_{\mathrm{zz}}-\mathrm{I}_{\mathrm{SO}}
$$

That Isoin equation (1) is Isotropic factor.

$$
\begin{aligned}
& \Delta \sigma=\sigma_{Z Z}-\frac{1}{2}\left(\sigma_{X X}+\sigma_{Y Y}\right) \\
& \eta=\frac{\sigma_{Y Y}-\sigma_{X X}}{\xi} \\
& \sigma_{v}^{i s o}=\sqrt{\frac{3}{2} \xi}(4) \\
& \sigma_{ \pm}=\frac{1}{2} \xi \\
& \Omega=\sigma_{33}-\sigma_{11}(6) \\
& \text { That } \Omega \text { in equation (6) is Span factor. } \\
& S_{k \sigma w}=\sigma_{Z Z}-\sigma_{X X}(7) \\
& K=\frac{3\left(1 s o-\sigma_{Y Y}\right)}{\Omega}(8)
\end{aligned}
$$

That $\Delta_{Y Y}$ in equation (8) can be substitute with $\Delta_{22}$ for gaining other $\mathrm{K}$ factor $[72,73]$.

\section{REFERENCES}

1. M. Monajjemi, and M. Ahmadianarog, Journal of Computational and Theoretical Nanoscience, 2014, 11(6), 1465-1471.

2. Reich, Eike; Anne Schibil, Valeria Widmer, Ruth Jorns, Evelyn Wolfram, Alison DeBatt Journal of Liquid Chromatography \& Related Technologies 2006, 29(14): 2141-2151,

3. Majid Monajjemi, Robert Wayne, Jrand James E. Boggs, Chemical Physics, 2014, 433,1-11.

4. Dattner, Christine; Boussabba, Sophie, Emmanuelle Javelle, ed., The Book of Green
Tea, Universe Books, 2003; 13

5. M. Monajjemi, M. Khosravi, B. Honarparvar International Journal of Quantum Chemistry, 2011, 111, 2771-2777.

6. A. Tahan and M. Monajjemi, ActaBiotheor 2011, 59: 291-312

7. Wang, L. F.; Zhang, H. Y. BioorgChem 2005, 3, 108.

8. Dulloo AG, Duret C, Rohrer D, Girardier L, Mensi N, Fathi M, Chantre P, Vandermander J Am. J. Clin. Nutr. 1999, 70(6): 1040-5,

9. Croft, K. D.; Ann, N. Y. Acad Sci 1998, 854, 
435.

10. F. Mollaamin, Z. Varmaghani, and M. Monajjemi, Physics and Chemistry of Liquids. 2011, 49(3), 318-336

11. Pietta, P. G. J Nat Prod 2000, 63, 1035.

12. M. Monajjemi , N. Karachi \& F. Mollaamin, Fullerenes, Nanotubes, and Carbon Nanostructures, 2014, 22: 643-662.

13. Shoeb, M. Bangladesh J Pharmacol, 2006, 1, 35.

14. Heiss, Mary Lou; Heiss, Robert J., The Story of Tea: A Cultural History and Drinking Guide, Ten Speed Press, 2007; 179-185

15. Evans, C. A.; Miller, N. J.; Paganga, G. Free RadicBiol Med 1996, 20, 933.

16. Maron DJ, Lu GP, Cai NS, et al. , Arch. Intern. Med. 2003, 163(12): 1448-53.

17. M. Monajjem, S. Ketabi, A. Amiri, Russian Journal of physical chemistry , 2006, 1, 5562.

18. Lambert JD, Sang S, Yang CS, Chem. Res. Toxicol. 2007, 20(4): 583-5,

19. Cabrera C, Artacho R, Gimenez R: Benefical effects of green tea: A review. J Am CollNutr 2006, 25: 79-99.

20. Sano M, Tabata M, Suzuki M, Degawa M, Miyase T, Maeda-Yamamoto M, Analyst 2001, 126: 816-820.

21. Willson KC: Coffee, Cocoa and Tea. New York: CABI Publishing, 1999.

22. Mckay DL, Blumberg JB, J Am CollNutr 2002, 21:1-13.

23. Hernandez, E. C.; Naranjo, A. R.; Luis, A.; Cabrera, M. J Mol Struct 2007, 819, 121.

24. Fatemeh Mollaamin and Majid Monajjemi, Physics and Chemistry of Liquids 2012, 50(5): 596-604

25. Monajjemi,M. Honarparvar, B. H. Haeri, H. Heshmat, M., Russian Journal of Physical Chemistry C., 2006, 80(1): S40-S44.

26. Nijveldt, R. J.; Nood, E. V.; Hoorn, D. E. C. V.; Boelens, P.G.; Norren, K.; Leeuwen, P. A. M. Am J ClinNutr 2001, 74, 418.

27. M.Monajjemi, S. Afsharnezhad, M.R. Jaafari, T.Abdolahi, A. Nikosade and Monajemi. Russian Journal of physical chemistry $A$, 2007, 2, 1956-1963.

28. Manach C, Scallbert A, Morand C, Remesy C, Jimenez L,Am JClinNutr 2004, 79: 727747.
29. Ostrowska, J.; Skrzydlewska, E. Adv Med Sci 2006, 51, 298.

30. MajidMonajjemi and A.Abedi, Research Journal of chemistry and Environment, 2006, 4, 37-41.

31. Wei, T.; Chen, C.; Hou, J.; Zhao, B.; Xin, W. Chin Sci Bull 2000, 45, 422.

32. Monajjemi M, Ghiasi R, Abedi A, Russian Journal of Inorganic Chemistry, 2005, 3, 382388.

33. Niemeyer, E. D.; Brodbelt, J.S. JAm Soc Mass Spectrom 2007, 18, 1749.

34. M. Monajjemi, A. Sobhanmanesh, \& F. Mollaamin, Fullerenes, Nanotubes, and Carbon Nanostructures, 2013, 21: 47-63.

35. Ogura, R.; Ikeda, N.; Yuki, K.; Morita, O.; Saigo, K.; Blackstock, C.; Nishiyama, N.; Kasamatsu, T. Food ChemToxicol 2008, 46, 2190.

36. B. Ghalandari, M. Monajjemi, and F. Mollaamin, Journal of Computational and Theoretical Nanoscience, 2011, 8, 1212 1219.

37. Seyoum, A.; Asres, K.; Fathy, K.; Fiky, E. Phytochemistry, 2006, 67, 2058.

38. M. Monajjemi, M. Khaleghian, J Clust Sci., 2011, 22, 673

39. Tang, D.; Sheng-Rong, S.; Xun, C.; Zhang, Y.; Chong, X. U.;Tang, J. Z. UnivSci 2004, 5, 6, 668.

40. H. Yahyaei\& M. Monajjemi, Fullerenes, Nanotubes, and Carbon Nanostructures, 2014, 22: 346-361.

41. Serge, A. J Mol Struct: Theochem 2008, 856, 38.

42. Cao G, Sofic E, Prior R, J Agric Food Chem 1996, 44: 3426-3431.

43. Langley-Evans S, Int J Food Sci Nutr 2000, 51:181-188.

44. Saffari Y, Sadrzadeh SMH, Life Sci 2004, 74: 1513-1518.

45. F. Mollaamin, K. Shahani pour, K. Shahani pour, A. R. Ilkhani, Z. Sheckari, and M.Monajjemi, Russian Chemical Bulletin, International Edition, 2012, 61(12), 21932198.

46. Golden, EB; Lam, P.Y.; Kardosh, A.; Gaffney, K. J.; Cadenas, E.; Louie, S. G.; Petasis, N. A.; Chen, T. C.; Schonthal, A. H., Blood 2009, 113 (23): 5927-37,

47. M. Monajjemi \& M. Falahati \& F. Mollaamin, 
Ionics 2013, 19,155-164

48. Zhang, M., International Journal of Cancer 2009, 124(6): 1404-8

49. Majid Monajjemi , Fatemeh Mollaamin, J Clust Sci 2012, 23: 259-272.

50. Sakane,, Kahori., Daily Yomiuri. The Buddhist Channel.Retrieved 2008, 2009-11-15.

51. M. Monajjemi , M. Heshmat ,H. H. Haeri, Biochemistry (Moscow), 2006, 71,, S113_S122.

52. Zhang MH, Luypaert J, Pierna JAF, Xu QS, MassartDL,Talanta 2004, 62, 25-35.

53. M.Monajjemi, M.H.Razavian, F.Mollaamin, F.Naderi, B.Honarparvar, Russian Journal of Physical Chemistry A 2008, 82(13), 22772285.

54. Mojzisova, G.; Kuchta, M. Physiol Res 2001, $50,529$.

55. M. Monajjemi, Struct Chem., 2012, 23, 551580 .

56. Knekt, P.; Kumpulainen, J.; Jarvinen, R.; Rissanen, H.; Heliovaara, M.; Reunanen, A.; Hakulinen, T.; Aromaa, A. Am J ClinNutr 2002, 76, 560.

57. F. Mollaamin , M. Monajjemi\& J. Mehrzad, Fullerenes, Nanotubes, and Carbon Nanostructures, 2014, 22: 738-751.

58. Havsteen, B. H. PharmacolTher 2002, 96, 67.

59. M. Monajjemi, M. Heshmat, H. H. Haeri, F. Kaveh, Russian Journal of Physical Chemistry. 2006, 80, 1061-1068.

60. Monajjemi, M., Lee, V. S., Khaleghian, M., Honarparvar, B., and Mollaamin, F., J. Phys. Chem. C., 2010, 114: 15315-15330.

61. T. Ardalan , P. Ardalan\& M. Monajjemi, Fullerenes, Nanotubes, and Carbon
Nanostructures, 2014, 22: 687-708.

62. Majid Monajjemi, Chemical Physics, 2013, 425, 29-45.

63. Monajjemi M, Ghiasi R, Ketabi S , Journal of Chemical Research-S 2004, 1: 11-1.

64. M. Monajjemi, J.E Boggs, J. Phys. Chem A. 2013, 117, 1670.

65. H. Yahyaei, M. Monajjemi, H. Aghaie, and K. Zare, Journal of Computational and Theoretical Nanoscience, 2013, 10(10) 2332-234.

66. M. Monajjemi, S. Ketabi, M. Hashemian Zadeh, and A. Amiri ,Biochemistry (Moscow), 2006, 71(1), S1-S8

67. Monajjemi M, Chahkandi B, J. Mol. Struct. (Theochem), 2004, 714: 43.

68. Monajjemi M, Ghiasi R, Sadjadi M.A.S, Applied Organometallic Chemistry 2003, 17 (8): 635-640.

69. M. Monajjemi, H. Baheri, and F. Mollaamin, Journal of Structural Chemistry. 2011, 52(1), 54-59.

70. F. Mollaamin, F. Najafi , M. Khaleghian , B. Khalili Hadad\& M Monajjemi, Fullerenes, Nanotubes, and Carbon Nanostructures, 2011, 19: 653-667.

71. M. Monajjemi , H. Yamola\& F. Mollaamin, Fullerenes, Nanotubes, and Carbon Nanostructures, 2014, 22: 595-603.

72. M. Monajjemi, E, Rajaeian, F. Mollamin, F.Naderi, S. Saki, Physics and Chemistry of Liquids., 2008, 46, 299-306.

73. M. Monajjemi, M. Heshmat, H. Aghaei, R. Ahmadi, K. Zare Bulletin of the Chemical Society of Ethiopia., 2007, 21, 111-116. 